\title{
$2+1$ flavour thermal studies on an anisotropic lattice
}

\author{
Chris Allton ${ }^{*, \dagger}$, Gert Aarts $^{a}$, Alessandro Amato ${ }^{a, b}$, Wynne Evans $^{a}$, Pietro Giudice ${ }^{c}$, \\ Timothy Harris $^{d}$, Simon Hands ${ }^{a}$, Aoife Kelly ${ }^{e}$, Sinéad M. Ryan ${ }^{d}$ and Jon-Ivar \\ Skullerud ${ }^{e}$ \\ a Department of Physics, College of Science, Swansea University, Swansea, United Kingdom \\ ${ }^{b}$ Institut für Theoretische Physik, Universität Regensburg, Regensburg, Germany \\ ${ }^{c}$ Universität Münster, Institut für Theoretische Physik, Münster, Germany \\ ${ }^{d}$ School of Mathematics, Trinity College, Dublin 2, Ireland \\ ${ }^{e}$ Department of Mathematical Physics, National University of Ireland Maynooth, Maynooth, \\ County Kildare, Ireland \\ ${ }^{\dagger}$ E-mail: c.allton@swan.ac.uk
}

The FASTSUM collaboration has initiated a detailed study of thermal QCD using $2+1$ flavours of improved Wilson quarks on anisotropic lattices. Spatial volumes of $(3 \mathrm{fm})^{3}$ and $(4 \mathrm{fm})^{3}$ are used at fixed cut-off with temperatures ranging from 120 to $350 \mathrm{MeV}$ (corresponding to temporal lattice extents of 48 to 16 lattice units). Results presented here include the deconfinement temperature and a study of the restoration of chiral symmetry, together with a brief summary of our collaboration's other results from these ensembles.

31 st International Symposium on Lattice Field Theory LATTICE 2013

July 29 August 3, 2013

Mainz, Germany

${ }^{*}$ Speaker. 


\section{Introduction}

Particle physics data are famously collated and summarised in the Particle Data Book [1]. However, it is interesting to note that there are no entries on the deconfined phase of QCD a symptom of the difficulty of studying (experimentally and theoretically) this new phase. The FASTSUM Collaboration has studied QCD at non-zero temperature for a number of years using dynamical quarks on anisotropic lattices where the temporal lattice spacing, $a_{\tau}$, is less than the spatial one, $a_{s}$. Since the temperature $T=1 /\left(a_{\tau} N_{\tau}\right)$, where $N_{\tau}$ is the number of lattice points in the temporal direction, this gives the distinct advantage that more points are sampled in a euclidean correlator for a given temperature compared to the isotropic case.

Our research programme began with two-flavour dynamical "1st generation" ensembles from which we studied a number of phenomenological quantities, such as spectral features in charmonium and bottomonium at zero and non-zero momenta, and the inter-quark potential in charmonium [2]. We have now improved the accuracy of our results by producing our " 2 nd generation" lattices which have $2+1$ dynamical flavours, a larger volume, improved discretisation and more realistic dynamical quark masses, see Table 1.

In this talk, I give an overview of our 2nd generation ensembles including our estimate of the deconfinement temperature, $T_{c}$, obtained from the Polyakov loop. I discuss the partial restoration of chiral symmetry in the light meson spectrum and briefly review four results obtained from these lattices which are covered fully in other talks $[3,4,5,6]$.

\section{Lattice details}

Our 2nd generation ensembles use the Hadron Spectrum Collaboration's (HSC) Symanzikimproved gauge action [8], with

$$
S_{G}=\frac{\beta}{\gamma_{g}}\left\{\sum_{x, s \neq s^{\prime}}\left[\frac{5}{6 u_{s}^{4}} \mathscr{P}_{s s^{\prime}}(x)-\frac{1}{12 u_{s}^{6}} \mathscr{R}_{s s^{\prime}}(x)\right]+\sum_{x, s} \gamma_{g}^{2}\left[\frac{4}{3 u_{s}^{2} u_{\tau}^{2}} \mathscr{P}_{s \tau}(x)-\frac{1}{12 u_{s}^{4} u_{\tau}^{2}} \mathscr{R}_{s \tau}(x)\right]\right\}
$$

where $\mathscr{P}$ and $\mathscr{R}$ are the usual $1 \times 1$ plaquette and $2 \times 1$ rectangular Wilson loops, $u_{s(\tau)}$ are the spatial (temporal) tadpole factors of the bare links, $\gamma_{g(f)}$ are the bare gauge (fermion) anisotropies and, as usual, $\beta=2 N_{c} / g^{2}$ and $N_{c}=3$ is the number of colours. The means of the stout-smeared links are $\tilde{u}_{\mu}$ (with $\tilde{u}_{\tau}=1$ ).

We use a tadpole-improved clover fermion action and stout-smeared links [9] using the same parameters as the Hadron Spectrum Collaboration [8],

$$
\begin{aligned}
S_{F} & =\sum_{x} \bar{\psi}(x) \frac{1}{\tilde{u}_{\tau}}\left\{\tilde{u}_{\tau} m_{0}+\gamma_{\tau} \nabla_{\tau}+\nabla_{\tau}^{2}+\frac{1}{\gamma_{f}} \sum_{s}\left[\gamma_{s} \nabla_{s}+\nabla_{s}^{2}\right]\right. \\
& \left.-\frac{1}{4}\left(\frac{\gamma_{g}}{\gamma_{f}}+\frac{1}{\xi}\right) \frac{1}{\tilde{u}_{\tau} \tilde{u}_{s}^{2}} \sum_{s} \sigma_{\tau s} F_{\tau s}+\frac{1}{2 \gamma_{f}} \frac{1}{\tilde{u}_{s}^{3}} \sum_{s<s^{\prime}} \sigma_{s s^{\prime}} F_{s s^{\prime}}\right\} \psi(x) .
\end{aligned}
$$

The first line is the usual Wilson action and the second line is the clover piece with $\tau$ and $s$ referring to temporal and spatial directions. The $\nabla_{\mu}$ are covariant finite differences and $\xi=a_{s} / a_{\tau}$ is the renormalised anisotropy. $\gamma_{s(\tau)}$ are the spatial (temporal) Dirac matrices and $\sigma_{\mu v}=\frac{1}{2}\left[\gamma_{\mu}, \gamma_{v}\right]$. 
We use the same parameters as the HSC employed in their studies [10] corresponding to an anisotropy, $\xi=3.5$. We generate ensembles with two volumes, $24^{3}$ and $32^{3}$, enabling us to study finite volume effects. We also make use of the $T=0$ (i.e. $N_{\tau}=128$ ) configurations kindly made available to us from HSC. Table 1 gives a full list of our parameters. The generation of the ensembles were performed using the Chroma software suite [11] with Bagel routines [12].

\section{Determination of the deconfining temperature}

The Polyakov loop, $L$, can be used to determine $T_{c}$ as follows [13]. We note that $L$ is related to the free energy, $F$, of a static quark, via:

$$
L(T)=e^{-F(T) / T} .
$$

However, $F$ is only defined up to an additive renormalisation constant, $\Delta F=f\left(\beta, m_{0}\right)$. We can impose a renormalisation condition at a renormalisation temperature, $T_{R}$, by requiring

$$
L_{R}\left(T_{R}\right) \equiv c
$$

for some suitable choice of $T_{R}$ and $c$. This means a multiplicative renormalisation constant, $Z_{L}$, can be fixed as follows

$$
L_{R}(T)=e^{-F_{R}(T) / T}=e^{-\left(F_{0}(T)+\Delta F\right) / T}=L_{0}(T) e^{-\Delta F / T}=L_{0}(T) Z_{L}^{N_{\tau}} .
$$

In Fig. 1, we plot the Polyakov loop with three different renormalisation schemes corresponding to different choices of $T_{R}$ and the constant in Eq. (3.2), as listed in the figure caption. By fitting the data to cubic splines we obtain the point of inflection $a_{\tau} T_{c}=0.0329(7)$ where the error reflects the spread from the three renormalisation schemes. This statistical uncertainty is given by the thickness of the three interpolating curves and can be seen to be negligible in this context. The result is then $N_{\tau}^{\text {crit }}=30.4(7)$ or $T_{c}=185(4) \mathrm{MeV}$.

\section{First results}

The deconfinement transition is expected to occur in the same $T$ range as the chiral symmetry restoration. For this reason it is interesting to study the chiral partners in the light meson sector to find evidence of this effect. In Fig. 2 we show the pseudoscalar and scalar meson correlator for $T / T_{c}=0.63$ and 1.90. As can be seen, in the high temperature case, these two channels are closer together than at low temperature illustrating the partial restoration of chiral symmetry in these quantities.

We have commenced studying several quantities on our 2 nd generation ensembles. Results on the following quantities have been reported in this conference and elsewhere.

- Susceptibility [3]. We study the electric charge susceptibility which is of interest experimentally to quantify fluctuations in heavy-ion collision experiments and for the determination of the electric charge diffusion coefficient. 


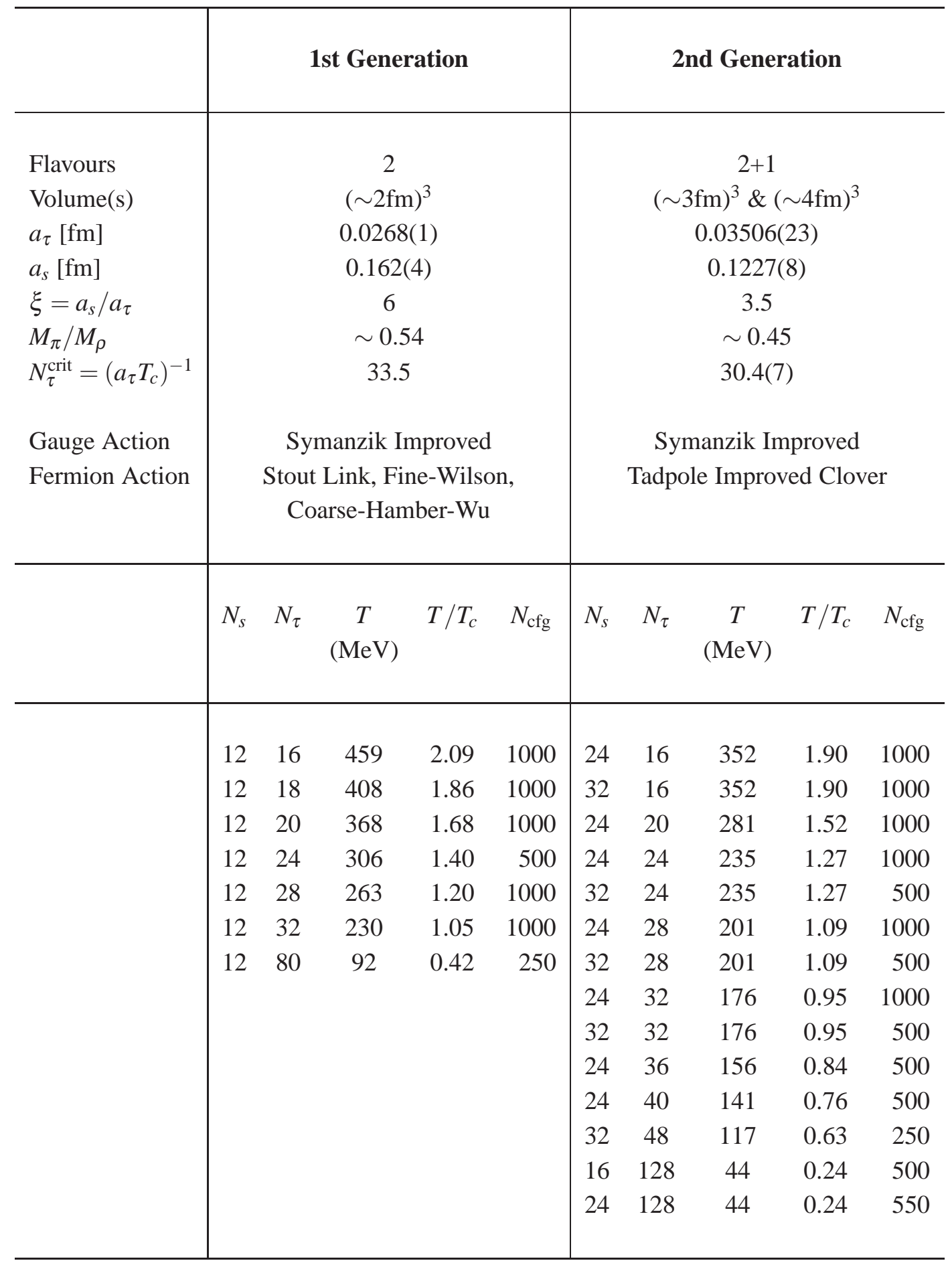

Table 1: A list of the lattice parameters used for our 1st and 2nd generation ensembles. 


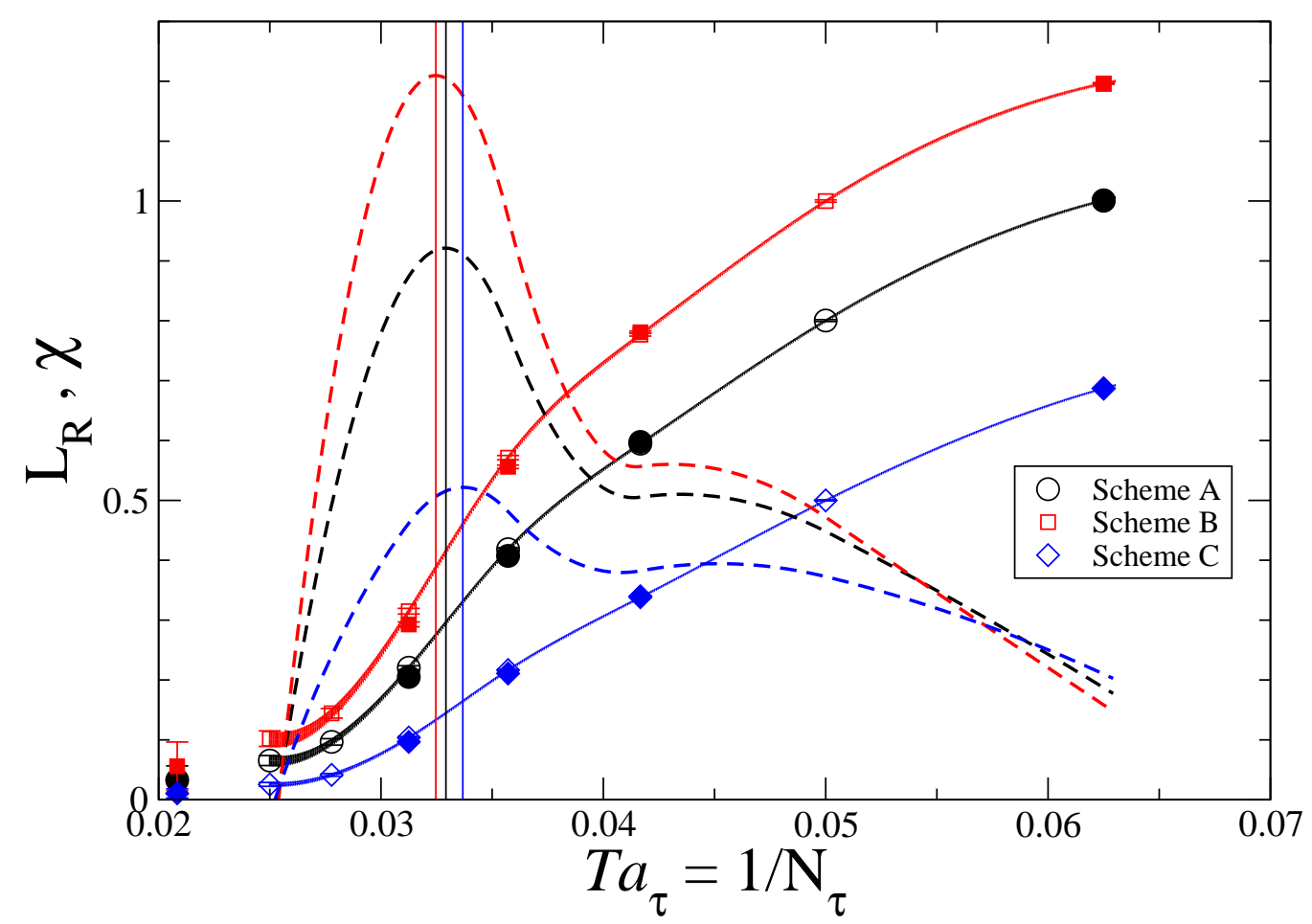

Figure 1: The renormalised Polyakov loop, $L_{R}$, depicted by solid $\left(32^{3}\right)$ and open $\left(24^{3}\right)$ symbols. The solid curves are obtained by cubic splines and their temperature derivatives, $\chi$, are depicted by dashed curves. Three renormalisation schemes are considered, Scheme A: $L_{R}\left(N_{\tau}=16\right)=1.0$, Scheme B: $L_{R}\left(N_{\tau}=20\right)=$ 1.0, Scheme C: $L_{R}\left(N_{\tau}=20\right)=0.5$.

- Electrical conductivity [4, 14]. The temperature dependence of the electrical conductivity has been calculated on our lattices, using the exactly conserved lattice current. We find that the conductivity divided by the temperature increases with temperature across the deconfinement transition. This is the first time this quantity has been computed as a function of temperature.

- Inter-quark potential in charmonium [5]. This is the first time this quantity has been calculated at high temperature with relativistic (rather than static) quarks. We find that its behaviour at low temperature agrees with the (confining) Cornell potential and that it becomes less confining as the temperature increases.

- Bottomonium spectrum [6]. We have used the NRQCD formulation to study spectral functions in bottomonium via the Maximum Entropy Method. We confirm our earlier result [2] that the S-wave $\left(\Upsilon\right.$ and $\left.\eta_{b}\right)$ ground states survive to $T \sim 2 T_{c}$ whereas excited states are suppressed, while the P-wave $\left(h_{b}, \chi_{b 0, b 1, b 2}\right)$ ground states dissociate close to $T_{c}$.

- Charmonium spectrum [7]. A study of charmonium spectral functions across the deconfining transition is also in progress. 


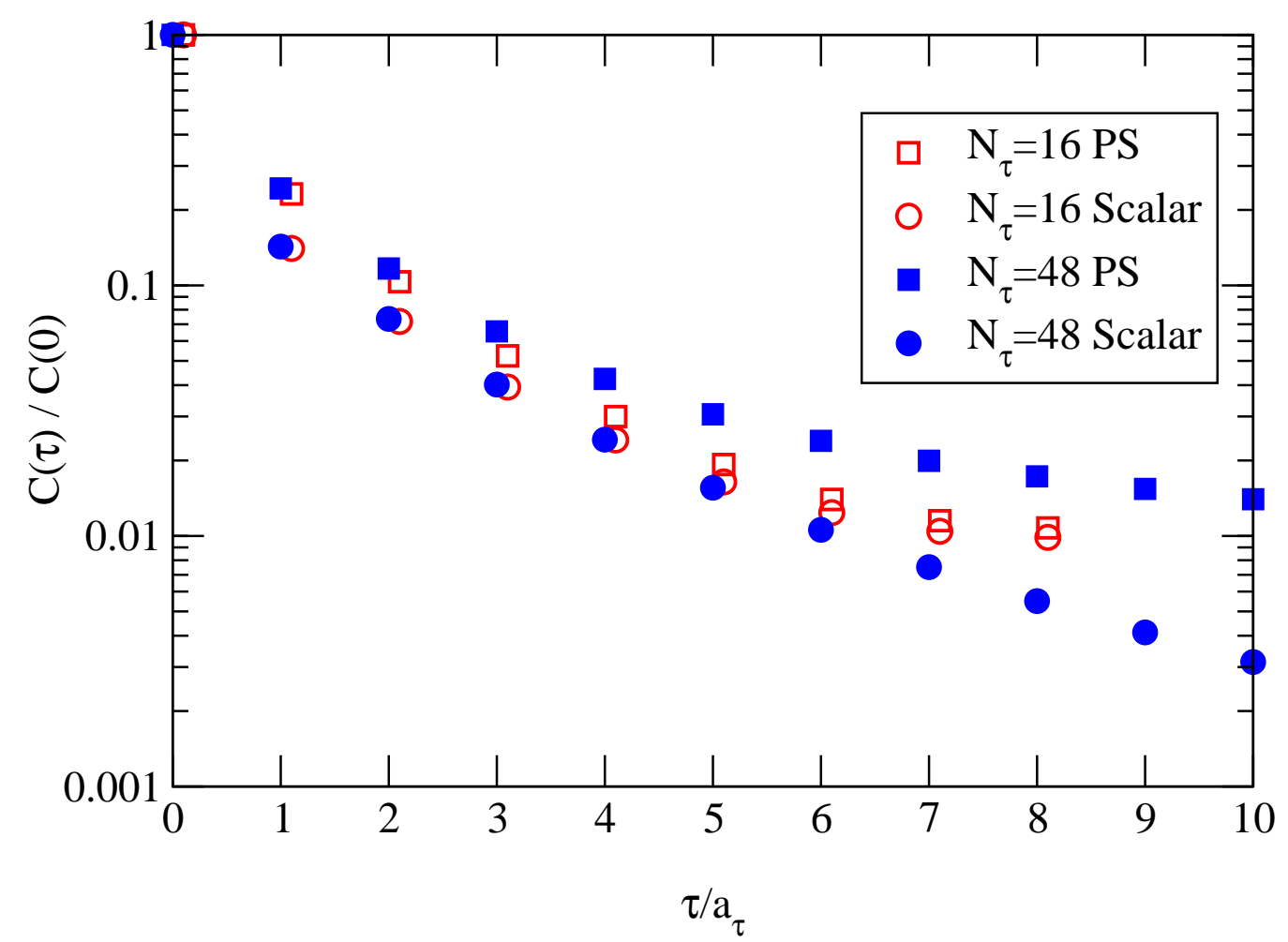

Figure 2: Correlation functions (normalised relative to $\tau=0$ ) for the light scalar and pseudoscalar mesons at two different temperatures on either side of the deconfinement transition, showing partial restoration of chiral symmetry. The $N_{\tau}=16$ points have been shifted horizontally for clarity.

\section{Conclusions}

This talk summarises our FASTSUM collaboration's latest finite-temperature studies using anisotropic lattices. We have improved upon our 1st generation 2 -flavour ensembles by generating ensembles which have 2+1 flavours, larger volume, improved discretisation, and smaller dynamical quark masses. In this talk, the deconfining temperature was presented and the (partial) chiral symmetry restoration in the light meson sector was studied. Other work presented elsewhere in this conference was summarised: the susceptibility, electrical conductivity, interquark potential in charmonium and (NRQCD) bottomonium spectral functions.

Our future plans are to improve our ensembles further - we are currently tuning our "3rd generation" ensembles which have a smaller temporal lattice spacing and have plans for a "4th generation" run with smaller spatial lattice spacing. We will thus be able to move towards a continuum extrapolation of all our quantities, leading to truly quantitative finite-temperature results for spectral quantities.

\section{Acknowledgements}

This work is undertaken as part of the UKQCD collaboration and the DiRAC Facility jointly funded by STFC, the Large Facilities Capital Fund of BIS and Swansea University. We acknowl- 
edge the PRACE Grants 2011040469 and Pra05_1129, European Union Grant Agreement No. 238353 (ITN STRONGnet), HPC Wales, the Irish Centre for High-End Computing, the Irish Research Council, the Leverhulme Trust, the Royal Society, the Science Foundation Ireland, STFC, and the Wolfson Foundation for support. The authors would like to thank Seyong Kim, Maria Paola Lombardo, Mike Peardon and Don Sinclair, for useful comments, discussions and collaboration.

\section{References}

[1] J. Beringer et al. [Particle Data Group Collaboration], Phys. Rev. D 86 (2012) 010001.

[2] G. Aarts, C. Allton, M. B. Oktay, M. Peardon and J. -I. Skullerud, Phys. Rev. D 76 (2007) 094513 [arXiv:0705.2198 [hep-lat]], M. B. Oktay and J. -I. Skullerud, arXiv:1005.1209 [hep-lat], G. Aarts, S. Kim, M. P. Lombardo, M. B. Oktay, S. M. Ryan, D. K. Sinclair and J. -I. Skullerud, Phys. Rev. Lett. 106 (2011) 061602 [arXiv:1010.3725 [hep-lat]], G. Aarts, C. Allton, S. Kim, M. P. Lombardo, M. B. Oktay, S. M. Ryan, D. K. Sinclair and J. I. Skullerud, JHEP 1111 (2011) 103 [arXiv:1109.4496 [hep-lat]], G. Aarts, C. Allton, S. Kim, M. P. Lombardo, M. B. Oktay, S. M. Ryan, D. K. Sinclair and J. -I. Skullerud, JHEP 1303 (2013) 084 [arXiv:1210.2903 [hep-lat]], P. W. M. Evans, C. R. Allton and J. -I. Skullerud, arXiv:1303.5331 [hep-lat], G. Aarts, C. Allton, S. Kim, M. P. Lombardo, S. M. Ryan and J. -I. Skullerud, arXiv:1310.5467 [hep-lat].

[3] P. Giudice, G. Aarts, C. Allton, A. Amato, S. Hands and J. -I. Skullerud, PoS(LATTICE 2013) 492, arXiv:1309.6253 [hep-lat].

[4] A. Amato, G. Aarts, C. Allton, P. Giudice, S. Hands and J. -I. Skullerud, PoS(LATTICE 2013) 176, arXiv:1310.7466 [hep-lat].

[5] P. W. M. Evans, C. Allton, P. Giudice and J. -I. Skullerud, PoS(LATTICE 2013) 168, arXiv:1309.3415 [hep-lat].

[6] T. Harris, S. M. Ryan, G. Aarts, C. Allton, S. Kim, M. P. Lombardo and J. -I. Skullerud, PoS(LATTICE 2013) 171, arXiv:1311.3208 [hep-lat].

[7] A. Kelly, "Spectral functions of charmonium in 2+1 flavour lattice QCD", poster at Strong and Electroweak Matter 2012, Swansea, 10-13 July 2012.

[8] H. -W. Lin et al. [Hadron Spectrum Collaboration], Phys. Rev. D 79 (2009) 034502 [arXiv:0810.3588 [hep-lat]].

[9] C. Morningstar and M. J. Peardon, Phys. Rev. D 69 (2004) 054501 [hep-lat/0311018].

[10] R. G. Edwards, B. Joo and H. -W. Lin, Phys. Rev. D 78 (2008) 054501 [arXiv:0803.3960 [hep-lat]].

[11] R. G. Edwards et al. [SciDAC and LHPC and UKQCD Collaborations], Nucl. Phys. Proc. Suppl. 140 (2005) 832 [hep-lat/0409003].

[12] P. A. Boyle, Computer Physics Communications v180, 12 (2009) 2739-2748.

[13] S. Borsanyi, Y. Delgado, S. Durr, Z. Fodor, S. D. Katz, S. Krieg, T. Lippert and D. Nogradi et al., Phys. Lett. B 713 (2012) 342 [arXiv:1204.4089 [hep-lat]].

[14] A. Amato, G. Aarts, C. Allton, P. Giudice, S. Hands and J. -I. Skullerud, Phys. Rev. Lett. 111 (2013) 172001 [arXiv:1307.6763 [hep-lat]]. 